\title{
Glucocerebrosidase gene-deficient mouse recapitulates Gaucher disease displaying cellular and molecular dysregulation beyond the macrophage
}

\author{
Pramod K. Mistry,b, , Jun Liu ${ }^{a}$, Mei Yang ${ }^{a}$, Timothy Nottolic, James McGrath ${ }^{a, d}$, Dhanpat Jain ${ }^{\mathrm{e}}$, Kate Zhang ${ }^{\mathrm{f}}$, \\ Joan Keutzer ${ }^{f}$, Wei-Lien Chuang ${ }^{f}$, Wajahat Z. Mehal ${ }^{b}$, Hongyu Zhaog, Aiping Lin ${ }^{g}$, Shrikant Mane ${ }^{\mathrm{h}}$, Xuan Liu', \\ Yuan Z. Pengi, Jian H. Li', Manasi Agrawali, Ling-Ling Zhu', Harry C. Blair ${ }^{j}$, Lisa J. Robinson ${ }^{j}$, Jameel Iqbali, Li Suni, \\ and Mone Zaidi
}

Departments of aPediatrics, ${ }^{b}$ Medicine, 'Comparative Medicine, dGenetics, and ePathology, Yale School of Medicine, New Haven, CT 06562; ${ }^{\mathrm{f}}$ Genzyme, Framingham, MA 02134; ${ }^{9}$ Yale School of Public Health and ${ }^{\text {h}}$ Keck Center for Genomics, Yale School of Medicine, New Haven, CT 06562; ${ }^{i}$ Mount Sinai Bone Program, Mount Sinai School of Medicine, New York, NY 10029; and ${ }^{\text {j} D e p a r t m e n t s ~ o f ~ P a t h o l o g y ~ a n d ~ C e l l ~ B i o l o g y, ~ U n i v e r s i t y ~ o f ~ P i t t s b u r g h ~ a n d ~ P i t t s b u r g h ~}$ Veterans Affairs Medical Center, Pittsburgh, PA 15261

Edited by Maria I. New, Mount Sinai School of Medicine, New York, NY, and approved September 10, 2010 (received for review March 19, 2010)

In nonneuronopathic type 1 Gaucher disease (GD1), mutations in the glucocerebrosidase gene (GBA1) gene result in glucocerebrosidase deficiency and the accumulation of its substrate, glucocerebroside (GL-1), in the lysosomes of mononuclear phagocytes. This prevailing macrophage-centric view, however, does not explain emerging aspects of the disease, including malignancy, autoimmune disease, Parkinson disease, and osteoporosis. We conditionally deleted the GBA1 gene in hematopoietic and mesenchymal cell lineages using an Mx1 promoter. Although this mouse fully recapitulated human GD1, cytokine measurements, microarray analysis, and cellular immunophenotyping together revealed widespread dysfunction not only of macrophages, but also of thymic T cells, dendritic cells, and osteoblasts. The severe osteoporosis was caused by a defect in osteoblastic bone formation arising from an inhibitory effect of the accumulated lipids LysoGL-1 and GL-1 on protein kinase C. This study provides direct evidence for the involvement in GD1 of multiple cell lineages, suggesting that cells other than macrophages may be worthwhile therapeutic targets.

osteopenia | osteoblast | glucosylsphingosine | T cells | PKC

M utations in the glucocerebrosidase gene (GBA1) in Gaucher disease (GD) lead to the accumulation of glucocerebroside (GL-1) in the lysosomes of mononuclear phagocytes and systemic infiltration by glycolipid-laden macrophages. The human GD1 phenotype is remarkable in its heterogeneity, and involves combinations of hepatosplenomegaly, bone marrow infiltration, cytopenia, skeletal defects, and pulmonary disease. The rare neuronopathic variants additionally display neurodegeneration (1). However, only part of the clinical variability of type 1 GD (GD1) can be attributed to GBA1 mutations. This discordance is exemplified by the striking variation in patients harboring identical mutations, between affected sibling pairs, and even in monozygotic twins (2).

The pathophysiologic steps that translate GBA1 deficiency and lysosomal GL-1 accumulation to the complex clinical phenotype are not known (3). The central paradox, however, is that, despite massive organomegaly, GL-1 accumulation accounts for less than $1 \%$ of organ weight, suggesting the involvement of additional interacting molecular mechanisms (3). Likewise, challenging the macrophagecentric view of GD1 is the recognition of unusual manifestations, such as gammopathies, cancer risk, pulmonary hypertension, cholesterol cholelithiasis, and Parkinson disease (3-7).

Although disabling skeletal complications, notably osteoporosis and fragility fractures, have been attributed to increased bone resorption, evidence for the involvement of osteoclasts in GD is weak. That osteoclasts have never been shown to harbor GL-1 storage material (8), that serum resorption markers are inconsistently elevated (9), and that potent inhibitors of bone resorption, namely the bisphosphonates, have modest, if any, effects in ameliorating the skeletal disease (10) all suggest that cell types beyond osteoclast precursors, or more generally, cells other than those of the mononuclear phagocyte lineage, might be affected.

Several groups have attempted to develop a mouse model that replicates GD1 fully (11). However, germline KO of the GBA1 gene results in neonatal lethality (11), whereas knocking-in disease mutations result in either unexpected lethality or mild increases in tissue GL-1, but without phenotypes that reflect clinical disease (12). The homozygous L444P knock-in mouse, for example, displays systemic inflammation, but no GL-1 accumulation (13). The recently described conditional GBA1 KO mouse, nonetheless, recapitulates the visceral manifestations of GD1, but not its skeletal defects (14).

Here, we report a mouse in which the GBA1 gene was deleted conditionally in cells of the hematopoietic and mesenchymal lineages through an Mx1 promoter (15). In addition to striking visceral and hematologic disease resembling human GD1, these GBA1-deficient mice (hence termed GBA1 mice) displayed profound osteopenia. We show that this osteopenia arises from a defect in bone formation as a result of the inhibition of PKC in osteoblasts by GL-1 and the minor lipid LysoGL-1. Furthermore, cytokine arrays, microarray, and immunophenotyping revealed not only the dysfunction of macrophages, but also striking, widespread aberrations in thymic T-cell and dendritic cell development. We believe that GBA1 mice are invaluable in delineating dysfunctional signaling pathways responsible for the clinical heterogeneity of human GD1.

\section{Results}

Conditional GBA1 Deletion. By conditionally deleting exons 8 through 11 of the GBA1 gene beginning at postnatal day 2, we achieved greater than $95 \%$ reduction of lysosomal glucocerebrosidase activity in cells of the hematopoietic and mesenchymal stem cell lineages in young adult 3- and 14-mo-old mice (Fig. S1 and SI Text). Consistent with the absence of GBA1, GBA1 mice exhibited a striking, up to approximately 60 - and approximately 30 -fold accumulation of GL-1 in liver (mean, approximately 15-fold) and spleen (mean, approxi-

Author contributions: P.K.M., J.L., M.Y., H.C.B., J.I., L.S., and M.Z. designed research; J.L, M.Y., T.N., J.M., D.J., KZ J K W.-LC, W. M., H.Z, A.L S.M X.L Y.P. J.H.L M.A., L.-L.Z., L.J.R. J.I., and L.S. performed research; P.K.M., W.-L.C., and M.Z. contributed new reagents/analytic tools; P.K.M., J.L., M.Y., T.N., J.M., D.J., K.Z., J.K., W.Z.M., H.Z., A.L., S.M., X.L., Y.P., J.H.L, L.-L.Z., H.C.B., L.J.R., J.I., L.S., and M.Z. analyzed data; and P.K.M., H.C.B., J.I., L.S., and M.Z. wrote the paper.

The authors declare no conflict of interest.

This article is a PNAS Direct Submission.

Data deposition: The data reported in this paper have been deposited in the Gene Ex pression Omnibus (GEO) database, www.ncbi.nlm.nih.gov/geo (accession no. GSE23086).

${ }^{1}$ To whom correspondence should be addressed. E-mail: pramod.mistry@yale.edu.

This article contains supporting information online at www.pnas.org/lookup/suppl/doi:10. 1073/pnas.1003308107/-/DCSupplemental. 
mately 10 -fold), respectively. There was also a dramatic and, importantly, an earlier elevation of LysoGL-1, a minor substrate, up to 166-fold (mean, approximately 30-fold) and 60-fold (mean, approximately 23 -fold) in liver and spleen, respectively (Fig. S1). The splenomegaly correlated with the tissue content of GL-1 and LysoGL-1, despite which the overall lipid content constituted no more than $1 \%$ of the weight of the grossly enlarged organs.

Visceral Phenotype of GBA1 Mice. GBA1 mice displayed exceptional runting and classic "gibbus" formation, seen typically in severe, type 3 GD (Fig. 1A) (1). They also exhibited hepatosplenomegaly, anemia, and a trend toward thrombocytopenia (Figs. $1 B-D$ ). Similar to human disease, GBA1 mice also showed low HDL cholesterol (4) and increased liver enzymes, but no increase in chitotriosidase, a serum biomarker of human GD1 (16) (Table S1). The latter finding was expected as, unlike the human chitotriosidase gene, the murine gene is not expressed in the mononuclear phagocytes. Noteworthy and reminiscent of human GD1 were the impressive, up to eightfold enlarged spleens, often with surface infarcts, as well as the distinct color and texture of the GBA1 mouse livers (Fig. $1 B$ and $C$ ).

The GBA1 phenotype was accompanied by a florid accumulation of storage cells in the liver, spleen, bone marrow, lymph nodes, and thymus (Fig. $1 E$, arrows). Estimates of the number of storage cells (as a percentage of total cells) were as follows: spleen, $50 \%$; liver, $30 \%$; lymph node, $20 \%$; thymus, $70 \%$. Transmission $\mathrm{EM}$ of the liver revealed storage cells harboring classic tubular structures (e.g., Fig. 1F). In addition, we found evidence for extramedullary hematopoiesis (Fig. $1 E$, Bottom) in both liver and spleen, which we believe can explain, at least in part, the massive organomegaly in GBA1 mice.

Immunophenotyping of GBA1 Mice. Not many studies have examined systematically how GBA1 mutations affect hematopoietic cells beyond those of the mononuclear phagocyte lineage. Only the L444P knock-in mouse has been reported to display features of inflammation, including B-cell proliferation and elevated IL$1 \beta$ and TNF $\alpha$ mRNA, but is without Gaucher cells or GL-1 accumulation (13). We immunophenotyped cells derived from the spleens, lymph nodes, and thymi of 6-mo-old GBA1 mice (Fig. 2). Compared with control littermates, GBA1 mice displayed increased cellularity in whole spleen $\left(14 \times 10^{7}\right.$ vs. $30 \times 10^{7}$ cells $)$, but not in thymi $\left(4.2 \times 10^{7}\right.$ vs. $\left.4.3 \times 10^{7}\right)$ or lymph nodes $(2.8 \times$ $10^{7}$ vs. $\left.2.3 \times 10^{7}\right)$. Overall, changes in cell populations in thymi were striking (Fig. 2), mostly with reciprocal changes in the spleen. The exceptions were the dendritic cell and macrophage populations, which were elevated both in thymi and spleens. Additionally, compared with control mice, GD1 thymi showed greater than 10-fold increase of $\mathrm{MHCII}^{+}$(IA/IE) and $\mathrm{B} 220^{+}$ (CD45R) cells (Fig. 2). In contrast, there was a reduction in the $\mathrm{CD} 4^{+} / \mathrm{CD}^{+}$cell population in GBA1 mice and an increase in the $\mathrm{CD}^{+}$cell subset (Fig. 2). Finally, cells bearing CD69, an early $\mathrm{T}$-cell activation marker, and CD44, an early thymocyte differentiation marker, were also increased in GBA1 mice compared with control mice (Fig. 2). Thus, thymic T-cell maturation was impaired in GBA1 deficiency.

To complement the flow cytometry studies, we measured cytokine levels in mouse serum using the Bioplex-23 cytokine array platform. Significant elevations of cytokines were noted
A

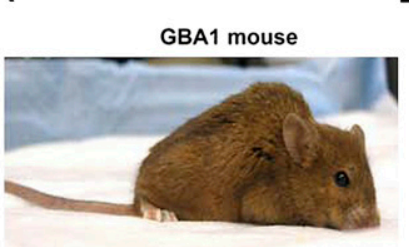

B

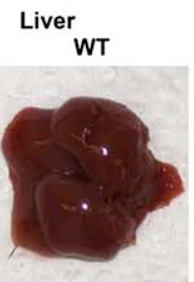

C

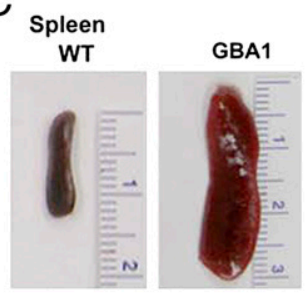

V

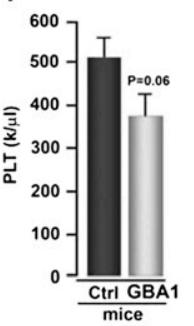

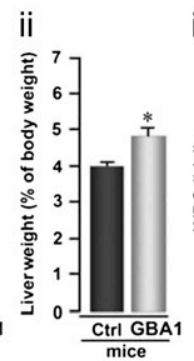

iii iv
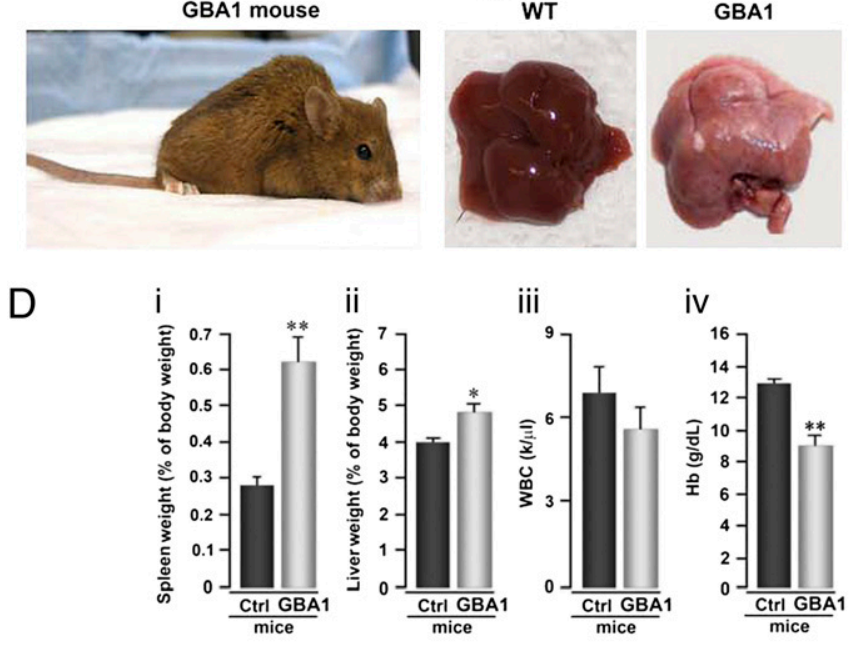

Spleen
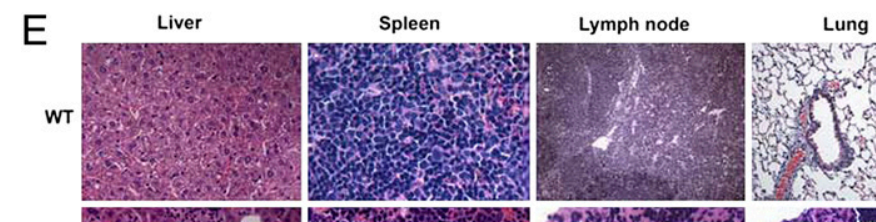

Lung
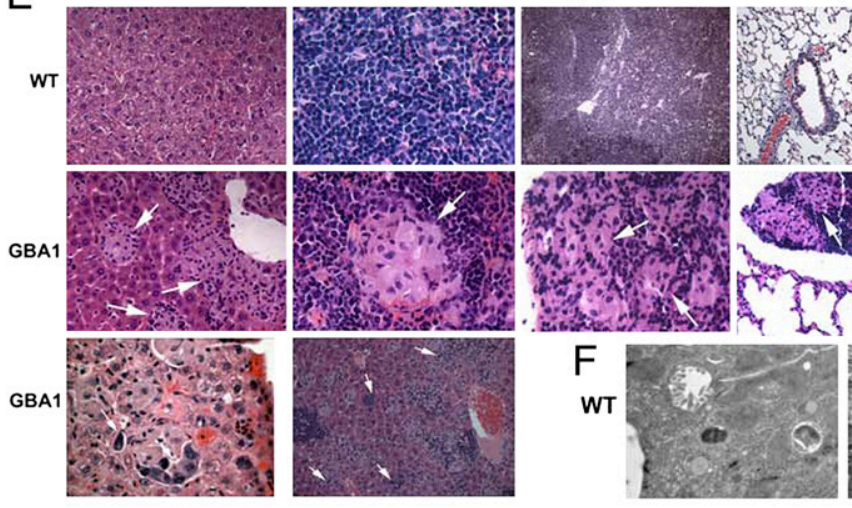

Thymus

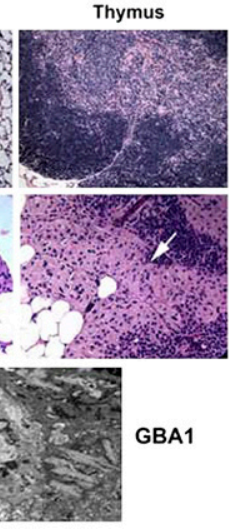

Fig. 1. GBA1 mice display a visceral and hematologic phenotype. (A) Gross appearance of a 14mo-old GBA1 mouse showing severe runting, Gibbus formation, and striking extremity pallor. (B) Hepatomegaly and (C) splenomegaly in GBA1 mice demonstrate areas of prominent surface infarction; note the difference in texture and color of the GBA1 organs. (D) Comparison of (i) spleen weight and (ii) liver weight as a percentage of body weight, as well as (iii) white blood cell (WBC) count, (iv) hemoglobin ( $\mathrm{Hb}$ ), and (v) platelet (PLT) count in GBA1 compared with control mice. Note the anemia and reduced platelet count. Student $t$ test, $* * P<0.01$, control versus GBA1, $n=19-59$ mice/group. (E) H\&E staining showing focal collections of classical foamy Gaucher cells (arrows) in two representative sections of liver, spleen, lymph nodes, lungs, and thymus of GBA1 mice. Control (WT) sections are shown. (E, Lower). Extensive extramedullary hematopoiesis in liver and spleens of GBA1 mice; arrows denote megakaryocytes. $(F)$ The extensive accumulation of tubular storage material within liver Gaucher cells was confirmed on transmission EM. 
A
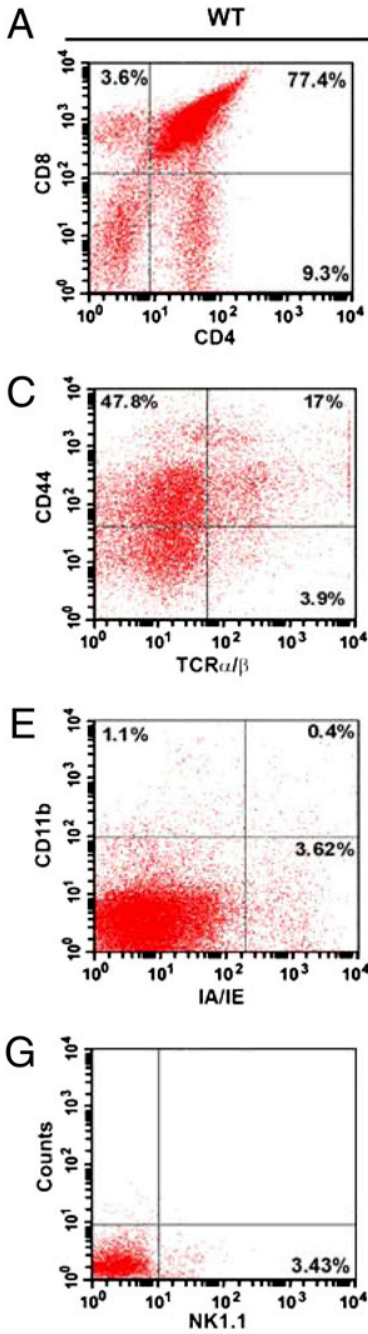

GBA1
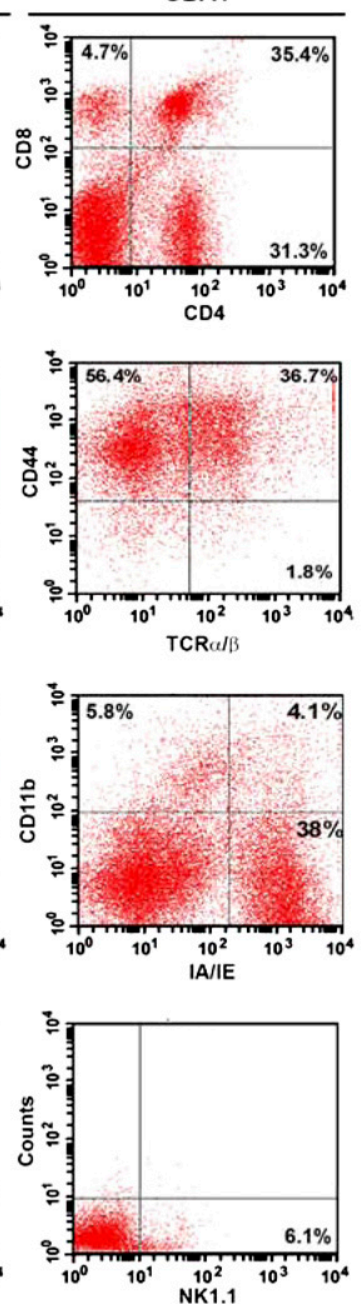

B
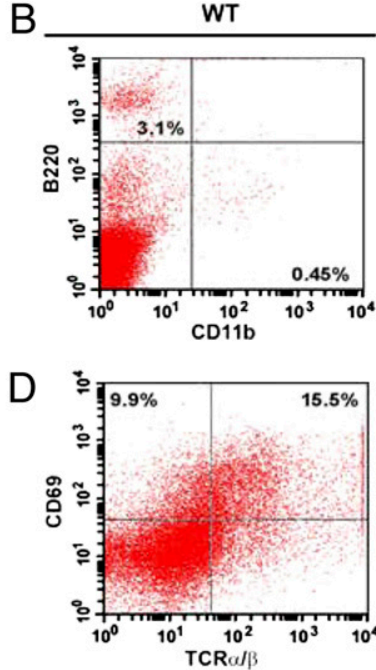

F
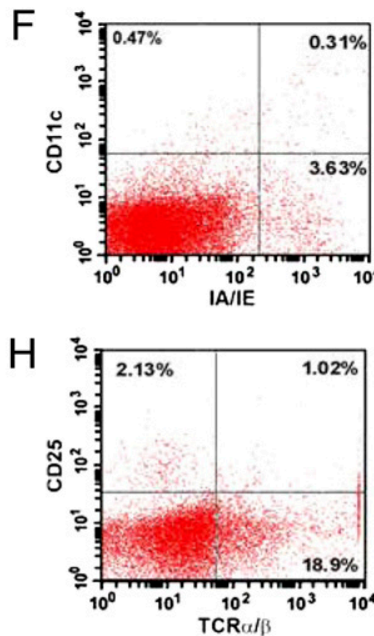

GBA1
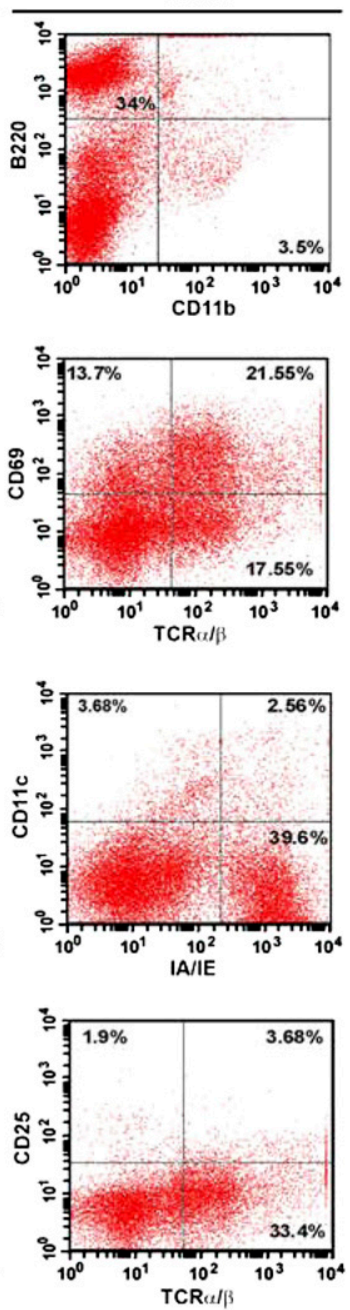

Fig. 2. Marked alternations in immune cell populations in the GBA1 mouse thymus. Thymocytes were isolated from 6-mo-old control (WT) and GBA1 mice and labeled with antibodies to CD4, CD8, CD11b, B220, TCR $\alpha / \beta$, CD44, CD69, IA/IE, CD11C, NK1.1, and CD25 for flow cytometry (Materials and Methods). Severely diseased GBA1 mice, selected as having a spleen size greater than five times the normal size, but having normal thymic weight and cellularity, were analyzed. Shown are representative data from three separate experiments.

(Table S2). Elevations in IL-1 $\beta$, IL-1 $\alpha$, IL-6, MIP-1 $\alpha$, MCP-1, and TNF- $\alpha$ were all consistent with increased macrophage numbers. Likewise, elevated IL-2, IL-10, GM-CSF, IFN- $\gamma$, IL-3, IL-9, MIP-1 $\beta$, and IL-13 are presumed to be derived from specific T-cell subsets likely involved in GD1 (Table S2).

Skeletal Phenotype of GBA1 Mice. The two most debilitating skeletal complications of human GD1 are focal osteonecrosis and osteopenia (1). Our GD1 mouse replicates both these features. Fig. $3 A$, ii shows histological evidence of medullary infarction and associated avascular osteonecrosis (cf. Fig. $3 A, i$ ). A mass of Gaucher cells is seen extruding from necrotic bone to form a focal mass of storage cells under the muscle layer, referred to as "Gaucheroma" in human disease (Fig. 3A, i). Additionally, typical clusters of abnormal Gaucher cells can be seen in the bone marrow of GBA1 mice (Fig. $3 A$, iii). Osteocyte lacunae in cortical bone were also grossly disorganized (cf. Fig. $3 A$, $i v$ and $v$ ).

Areal bone mineral density (BMD) measurements showed that 14-mo-old GBA1 mice had significant osteopenia at all sites, namely the lumbar spine, femurs, and tibiae (Fig. $3 B$ ). Micro-CT of trabecular bone of the lumbar spine showed a reduction in trabecular bone volume (BV/TV) and a trend toward decreased trabecular number and thickness with increased trabecular spacing (Fig. $3 C$ and $D$ ). This pattern of bone loss was confirmed on static histomorphometry (Table S3), but statistical significance could not be achieved because of the anisotropic, regionally nonuniform effect of GD1, in part reflecting local tumor-like effects and necrosis, but, in part, also reflecting focal effects of Gaucher cells on bone formation.

Dynamic histomorphometry performed following calcein labeling showed a significant $(P=0.036)$ reduction in bone formation rate in 14-mo-old GBA1 mice compared with control littermates (Table 1). This effect was not seen in younger 3-mo-old, immediately postpubertal, mice (Table S4). The latter result suggests that the depletion of GBA1 beginning postnatal day 2 did not affect bone formation, and hence, bone acquisition during growth. TRAP-labeled surfaces, indicative of the rate of bone resorption, remained unaltered at both ages (Table 1 and Table S4).

Cellular and Molecular Basis of Osteoblast Defect. We examined the cellular basis of this dramatic reduction in bone formation by $(i)$ measuring the proliferation of bone marrow stromal cells by MTT assay, (ii) examining osteoblast colony formation at day 10 and day 21 in ex vivo stromal cell cultures, and (iii) quantitating osteoblast gene expression by quantitative PCR in 10-d cultures.

The proliferation of isolated stromal cells was attenuated significantly in GBA1 ex vivo cultures compared with controls, but this reduction was restored to control levels by the addition of 
Fig. 3. Bone marrow infiltration, avascular necrosis, and systemic osteopenia in GBA1 mice. $(A)$ Control vertebral section with adjacent bone marrow (i) shows marked differences fromGBA1 mice (ii), which have focal collections of Gaucher cells (arrow) interspersed within the normal vertebral hematopoietic marrow. (iii) GBA1 mouse bone section showing medullary infarction and avascular necrosis; also shown is an extrusion of Gaucher cells into skeletal muscle. Control section (iv) of femoral cortical bone compared with a GBA1 section ( $v$ ) showing disorganized osteocyte lacunae with a loss of lamellar structure. (B) Areal BMD (aBMD) using a Lunar Piximus densitometer in 14-mo-old GBA1 and control (WT) mice at six sites, namely lumbar spine (L4-L6 and $>\mathrm{L} 6$ ) and right and left femurs and tibiae as shown. Student $t$ test was used to compare GD1 and WT mice; $n=2-10$ mice/group; ${ }^{*}<$ $0.05, * * P<0.01$. (C and $D$ ) Micro-CT of lumbar spine shows representative images of lumbar vertebrae, as well as the mean and range (parentheses) of BV/TV, trabecular number (Tb.N), trabecular thickness (Tb.Th.), and trabecular spacing (Tb.Sp.) in GBA1 mice compared with WT littermates $(n=2$ 10 mice/group). The orange color in the composite images shows a reduction in mineralized bone $(D)$. MTT assay $(E)$ shows proliferation of bone marrow stromal cells from WT and GBA1 mice with or without PMA. ( $F$ and $G$ ) ALP-positive cfu- $f$ and $(H)$ von Kossapositive cfu-ob from WT and GBA1 mice with or without PMA. Results are shown as optical density (OD) $(E)$, or cfu-f (\%); mean \pm SEM, or representative wells. Statistics by Student $t$ test: ${ }^{*} P<0.01$; four mice per group, triplicate estimations for all three experiments. (I) Markers of osteoblast differentiation, namely ALP, BSP, Runx2, and osterix by quantitative PCR in GBA1 mice compared with WT. Student $t$ test was used to compare WT and GBA1 mice; $n=2-10$ mice in triplicate wells; $* P<0$. 05 , ** $P<0$. 01 . $(J)$ Ex vivo TRAP-positive osteoclast formation in WT and GBA1 mice. Student $t$ test was used to compare WT and GBA1; $n=2-10$ mice per group, eight wells per group; $P>0.5$.

phorbol 12-myristate 13-acetate (PMA), a PKC activator (Fig. $3 E$ ). In addition, alkaline phosphatase-positive cfu-fibroblast (cfuf) colony formation was attenuated in GD1 cultures (Fig. 3F), but this reduction was again restored by PMA (Fig. $3 G)$. Late osteoblast differentiation, notably von Kossa-positive cfu-osteoblastoid (cfu-ob) formation, was modestly reduced in GBA1 ex vivo cultures compared with controls, but was enhanced by PMA (Fig. $3 H$ ). Reduced osteoblast differentiation in GBA1 mice was confirmed by a marked reduction in alkaline phosphatase (ALP), bone sialoprotein (BSP), Runx2, and osterix mRNA expression (Fig. 3I). Together, the findings suggest that the inhibitory effect of GBA1 deficiency on both precursor proliferation and early differentiation are mediated, at least in part, through PKC.

That an osteoblast phenotype was prominent in GBA1 mice led us to examine if it could be secondary to altered osteoclastogenesis. However, the static bone histomorphometry parameters suggested that there was no augmentation of in vivo osteoclast activity (Table S4). Consistent with this, ex vivo osteoclast formation was normal in cultures from 14-mo-old mice (Fig. $3 J$ ).

Two lines of evidence suggest that defective PKC activation contributes, at least in part, to the osteoblast defect of GBA1 deficiency. First, as noted earlier, the attenuated proliferation and differentiation of GBA1-deficient osteoblasts were both fully restored by PMA, a PKC activator. Second, the lipids GL-1 and LysoGL-1 that accumulate in GBA1-deficient cells are known to modulate PKC; in particular, LysoGL-1 inhibits PKC activation (24).

We therefore determined whether the PMA-induced osteoblast precursor proliferation and/or differentiation were sensitive to inhibition by GL-1 and/or LysoGL-1. In bone marrow stromal cell cultures, LysoGL-1, but not GL-1, inhibited PMA-induced precursor proliferation (Fig. $4 A$ ), as well as cfu-f formation (Fig. $4 B$ ). The inhibition of PMA-induced cfu-ob formation was relatively modest (Fig. 4C). Quantitative PCR confirmed the inhibition of the PMA-induced osteoblast differentiation genes Runx2 and BSP (Fig. 4D). In confirmatory experiments using MC3T3, E1 osteoblast precursor cultures also showed strong inhibition byLysoGL-1 (but not GL-1) of PMA-induced CFU-f formation, and ALP and Runx2 mRNA expression (Fig. 4E). Thus, we suggest that the minor lipid LysoGL-1 and, to an extent, GL-1 inhibit PKC-mediated osteoblast proliferation and early differentiation, and thus likely contribute to the bone formation defect in GBA1 mice. 
Table 1. Histomorphometry parameters, including osteoclast surfaces, measured in 14-mo-old GBA1 and control (WT) mice

\begin{tabular}{lccc} 
Parameter & WT $(n=4)$ & GBA1 mice $(n=8)$ & $P$ value* \\
\hline Bone forming/total area, calcein labeled/total area & $0.175 \pm 0.017$ & $0.136 \pm 0.007$ & 0.022 \\
Mineral apposition rate, $\mu \mathrm{m} / \mathrm{d}$ & $2.28 \pm 0.1$ & $2.772 \pm 0.08$ & NS \\
Osteoclast surface, TRAP label/total area & $0.161 \pm 0.055$ & $0.182 \pm 0.024$ & NS \\
Bone forming rate, $\mu \mathrm{m}^{3} / \mathrm{mm}^{2}$ & $494 \pm 50$ & $377 \pm 29$ & 0.0361 \\
\hline
\end{tabular}

Mean number of mice \pm SEM shown. NS, not significant.

*Student $t$ test.

Microarray Profiling of GBA1 Mice. Using an Affymetrix Gene 1.0 ST Array displaying 28, 869 genes, we compared control gene expression patterns against successively increasing severities of the GD1 phenotype, i.e., no clinical phenotype, and mild, moderate, and severe (i.e., life-threatening) GD1. Genes whose expression altered sequentially in relation to disease severity were identified [Table S5; Gene Expression Omnibus (GEO) accession no. GSE23086]. For biomarker studies, spleen and liver datasets were sorted into three groups per disease severity. Ingenuity profiling showed that certain proteases, including cathepsins $\mathrm{S}$ and $\mathrm{Z}$, and MMP-12 (all of which are involved in tissue remodeling) are exclusive markers of severe disease (Table S6). Conversely, the normal group consisted of candidate biomarkers that were elevated in a nondisease state, but in the presence of mild or severe disease, the genes were down-regulated. Thus, the latter genes could potentially be used to exclude GD1. Similarly, candidate genes for moderate and severe disease could be used to easily identify disease severity (Table S6).

To our knowledge, an exploration of gene expression in the viscera of murine GD1 models has not been reported. We show that, in the liver and spleen, 291 and 89 genes, respectively, displayed a 5- to 50-fold elevation in transcript levels, whereas considerably fewer genes were down-regulated (Table S7). Although the pathophysiologic relevance remains unclear, a dramatic up-regulation of several peptidases, lipases, and amylases was noted in the spleen, whereas in the liver a number of proteases, such as MMPs and cathepsins, were up-regulated by at least 20 -fold. Finally, genes in- volved in cell cycle, immune response, and signal transduction were up-regulated as a function of disease severity.

\section{Discussion}

The hallmark of GD1 is the tissue macrophage engorged with GL1-containing lysosomes (1). Pathophysiologic delineation and therapeutic intervention has therefore focused on this single cell type, the macrophage. This approach is testified by the success of macrophage-directed enzyme therapy (1). However, poorly responsive variants of GD1, such as cancers, Parkinson disease, hepatocellular disease, and osteoporosis, have emerged. This clinical complexity seen with a single gene defect, in essence, underscores our limited understanding of the multiple cell types and pathways that are likely involved in the pathogenesis of GD1 (17). Here, we report that a mouse in which the GBA1 gene was deleted conditionally using an Mx1 promoter recapitulated the human disease almost in its entirety. In addition, we noted hitherto unexpected effects on T-cell and dendritic cell development and osteoblastic bone formation.

The dramatic reduction in bone formation, which we show is caused by a defect in osteoblastogenesis, was importantly not accompanied by increased bone resorption. This observation is consistent with the limited efficacy of macrophage-targeted therapies in reversing osteopenia (10). It is also in agreement with the increased GL-1 content and abnormal secretome in bone marrow stromal cells cultured from a GD1 patient (18). Mechanistically, it could be explained, at least in part, by the direct in-
A
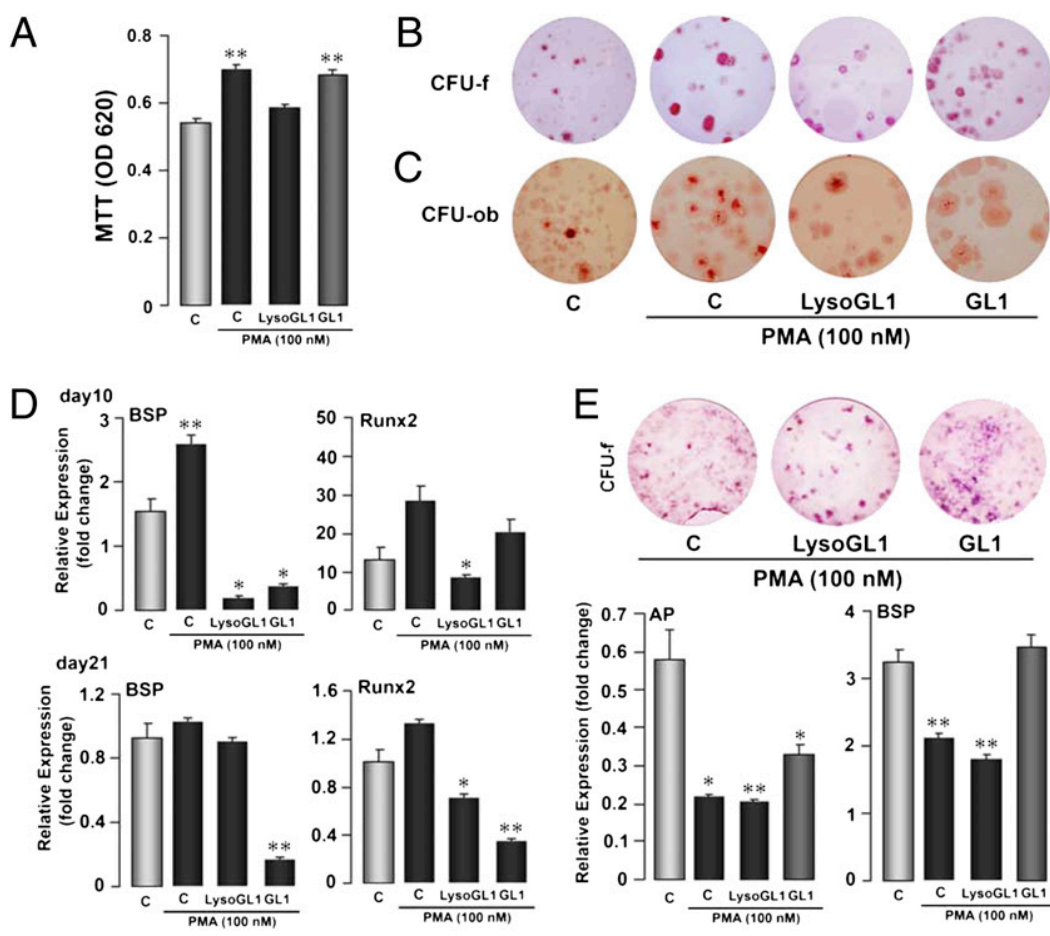

E
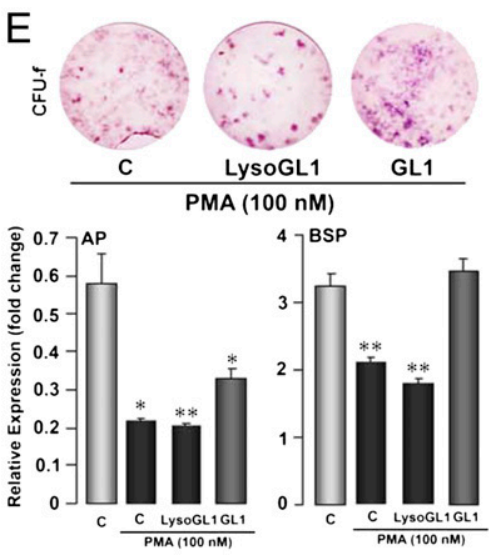

Fig. 4. LysoGL-1 inhibits osteoblastogenesis via PKC. (A) Proliferation of bone marrow stromal cells in the presence of PMA $(100 \mathrm{nM})$ with or without LysoGL-1 $(10 \mu \mathrm{M})$ or GL-1 $(40 \mu \mathrm{M})$, using the MTT assay. ( $B$ and $C$ ) ALP-positive cfu-f $(B)$ or cfu-ob $(C)$ with or without PMA and LysoGL-1 or GL-1. Results are shown as optical density (OD) $(A)$ or representative wells ( $B$ and $C$ ). ( $D$ ) Markers of osteoblast differentiation, namely BSP and Runx2, for 10- and 21-d cultures by quantitative PCR in the presence of PMA with or without Lyso-GL1 or GL-1. Statistics by Student $t$ test: ${ }^{*} P<0.01$ vs. zero dose; $n=4$ mice per group, triplicate estimations for all experiments. (E) Differentiation ofMC3T3. E1 osteoblast precursors, assessed by cfu-f formation and quantitative PCR for ALP and Runx 2 (at day 10) with or without PMA and LysoGL-1 or GL-1. Results are shown as representative wells or relative expression. Statistics by Student $t$ test: $* * P<0.01$ vs. zero dose, triplicate estimations. 
hibition of osteoblastogenesis by the lipids LysoGL-1 and GL-1. Overall, it appears that the osteoblast defect is distinct and autonomous. Nonetheless, poor osteoclast-osteoblast signaling from Gaucher osteoclasts, for example, via reduced sphingosine1-phosphate production $(19,20)$, may also play a causal role.

That osteoblastic bone formation is defective in GD1 opens the possibility of the use of a skeletal anabolic agent. Moreover, it is possible that non-cell-specific small molecules, such as substrate inhibitors, might be more efficacious in reversing GD1related osteopenia than macrophage-directed therapies. Indeed, the improvements of BMD with substrate inhibition have not previously been seen with macrophage-directed therapy $(28,29)$.

Although the complex clinical profile of human GD1 includes an increased risk for cancers, gammopathies, autoimmune disease, and infections, there is paucity of studies that have examined the effects of human GBA1 deficiency on the immune system. Our GBA1 mouse has allowed an in-depth analysis of the Tand B-cell, macrophage, and dendritic cell repertoire. We show a widespread effect of GBA1 deficiency on various immune cell populations. Most notably, thymic T-cell development was profoundly impaired, with reciprocal changes in the spleen. Corresponding changes in serum cytokine levels indicated global effects on both innate and adaptive immune systems. Finally, there was widespread extramedullary hematopoiesis in both spleen and liver, suggesting bone marrow failure. As accumulated GL-1 and LysoGL-1 constitute no more than $1 \%$ of the weight of the grossly enlarged organs, we attribute organomegaly at least in part to the striking extramedullary hematopoiesis.

The mechanism of the widespread immune dysfunction remains unclear. Could it be similar to what we demonstrated in the osteoblast, in which inhibition of osteoblastogenesis occurs through LysoGL-1 and GL-1 interactions with PKC? Indeed, GL-1 and LysoGL-1 have opposing effects, respectively, in stimulating and inhibiting PKC activation $(24,25)$. Connectivity mapping on our microarray data shows an overall reduction in PKC activation, suggesting that the minor lipid LysoGL-1 may be more important than previously appreciated. LysoGL-1 also interacts with its orphan $\mathrm{G}$ protein-coupled receptor, the T-cell death-associated gene 8 (TDAG8), to induce multinucleate

1. Grabowski GA (2008) Phenotype, diagnosis, and treatment of Gaucher's disease. Lancet 372:1263-1271.

2. Sidransky E (2004) Gaucher disease: Complexity in a "simple" disorder. Mol Genet Metab 83:6-15.

3. Cox TM (2001) Gaucher disease: Understanding the molecular pathogenesis of sphingolipidoses. J Inherit Metab Dis 24(Suppl 2):106-121.

4. Taddei TH, et al. (2009) The underrecognized progressive nature of N370S Gaucher disease and assessment of cancer risk in 403 patients. Am J Hematol 84:208-214.

5. Mistry PK, et al. (2002) Pulmonary hypertension in type 1 Gaucher's disease: genetic and epigenetic determinants of phenotype and response to therapy. Mol Genet Metab 77:91-98.

6. Taddei T, et al. (2010) High incidence of cholesterol gallstones in Gaucher disease Characterizing the biliary phenotype of type 1 Gaucher disease. $J$ Inherit Metab Dis 33:291-300.

7. Sidransky E (2005) Gaucher disease and parkinsonism. Mol Genet Metab 84:302-304

8. Elstein D, Itzchaki M, Mankin HJ (1997) Skeletal involvement in Gaucher's disease. Baillieres Clin Haematol 10:793-816

9. Sims KB, et al. (2008) Improvement of bone disease by imiglucerase (Cerezyme) therapy in patients with skeletal manifestations of type 1 Gaucher disease: Results of a 48-month longitudinal cohort study. Clin Genet 73:430-440.

10. Wenstrup RJ, et al. (2004) Gaucher disease: Alendronate disodium improves bone mineral density in adults receiving enzyme therapy. Blood 104:1253-1257.

11. Sidransky E, Ginns El (1997) Gaucher's disease: The best laid schemes of mice and men. Baillieres Clin Haematol 10:725-737.

12. Xu YH, Quinn B, Witte D, Grabowski GA (2003) Viable mouse models of acid betaglucosidase deficiency: The defect in Gaucher disease. Am J Pathol 163:2093-2101.

13. Mizukami $\mathrm{H}$, et al. (2002) Systemic inflammation in glucocerebrosidase-deficient mice with minimal glucosylceramide storage. J Clin Invest 109:1215-1221.

14. Enquist IB, et al. (2006) Effective cell and gene therapy in a murine model of Gaucher disease. Proc Natl Acad Sci USA 103:13819-13824. globoid cells. These cells characterize yet another lysosomal disease, Krabbe disease (25).

Thus, although dysfunctional signaling pathways in GD1 remain to be delineated precisely, our study provides evidence for an effect of GBA1 deficiency on cell lineages other than mononuclear phagocytes-most notably osteoblasts, dendritic cells, and $\mathrm{T}$ cells - in the pathophysiology of the diverse and complex clinical spectrum of GD1. These findings, we believe, pave the way for investigations that would define the role of each cell type, and the mechanism of their pathogenetic contribution(s), by genetically ablating affected cells lineages on a GBA1 background.

\section{Materials and Methods}

All procedures involving animals were reviewed and approved by In stitutional Animal Care and Use Committees of Yale School of Medicine and Mount Sinai School of Medicine (SI Text).

For immunophenotyping, cells were stained with appropriate antibodies, namely anti-CD4-FITC, anti-CD11b-FITC, anti-CD44-PE, anti-CD25-PE, antiCD45R (B220)-PE, anti-IA/IE-PE (BD Biosciences), anti-CD8-PerCP-Cy5.5, antiCD69-PE, anti-CD11c-PerCP-Cy5.5, anti-CD11b-PerCP-Cy5.5 (eBiosciences), and anti-TCR $\alpha \beta$-APC (Accurate Chemical). Flow cytometry was performed on a FACSCalibur device (Beckton Dickinson) and data analyzed using WinMDI 2.8 software.

Dual-energy absorptiometry was used to measure BMD in anesthetized mice by a Lunar Piximus densitometer (GE Medical Systems). Dissected vertebrae were also subjected to micro-CT using a Viva 40 system (Scanco). Bone formation and resorption were quantitated by histomorphometry following two sequential injections of calcein $(15 \mathrm{mg} / \mathrm{kg}) 5 \mathrm{~d}$ apart before euthanasia. Bone marrow cell cultures were performed for the assessment of osteoblast and osteoclast formation, using established protocols for cfu-f and TRAPpositive osteoclast formation, respectively (26). Quantitative PCR was performed as described (26).

ACKNOWLEDGMENTS. P.K.M is supported by National Institute of Diabetes Digestive and Kidney Diseases Grant K24DK066306, a Gaucher Generation Program grant, and a grant from the National Gaucher Foundation. M.Z. and L.S. are supported by National Institutes of Health Grants AG23176 DK80490, and DK70526. J.I. was supported by the American Federation of Aging Research.

15. Kühn R, Schwenk F, Aguet M, Rajewsky K (1995) Inducible gene targeting in mice Science 269:1427-1429.

16. Aerts JM, et al. (2008) Biomarkers for lysosomal storage disorders: Identification and application as exemplified by chitotriosidase in Gaucher disease. Acta Paediatr Supp/ 97:7-14.

17. Elleder M (2006) Glucosylceramide transfer from lysosomes-the missing link in molecular pathology of glucosylceramidase deficiency: A hypothesis based on existing data. J Inherit Metab Dis 29:707-715.

18. Campeau PM, et al. (2009) Characterization of Gaucher disease bone marrow mesenchymal stromal cells reveals an altered inflammatory secretome. Blood 114 3181-3190.

19. Ryu J, et al. (2006) Sphingosine 1-phosphate as a regulator of osteoclast differentiation and osteoclast-osteoblast coupling. EMBO J 25:5840-5851.

20. Ginzburg L, Kacher Y, Futerman AH (2004) The pathogenesis of glycosphingolipid storage disorders. Semin Cell Dev Biol 15:417-431.

21. Lukina E, et al. (2010) A phase 2 study of eliglustat tartrate (Genz-112638), an oral substrate reduction therapy for Gaucher disease type 1. Blood 116:893-899.

22. Pastores GM, Elstein D, Hrebícek M, Zimran A (2007) Effect of miglustat on bone disease in adults with type 1 Gaucher disease: A pooled analysis of three multinational, openlabel studies. Clin Ther 29:1645-1654.

23. Hannun YA, Bell RM (1989) Functions of sphingolipids and sphingolipid breakdown products in cellular regulation. Science 243:500-507.

24. Shayman JA, et al. (1991) Modulation of renal epithelial cell growth by glucosylceramide. Association with protein kinase C, sphingosine, and diacylglycerol. J Biol Chem 266 22968-22974.

25. Im DS, Heise CE, Nguyen T, O'Dowd BF, Lynch KR (2001) Identification of a molecular target of psychosine and its role in globoid cell formation. J Cell Biol 153:429-434.

26. Sun L, et al. (2006) FSH directly regulates bone mass. Cell 125:247-260. 